\title{
Optimización del consumo de disolvente Lule 10 en un proceso litográfico a partir de la metodología DMAMC de programas seis sigma
}

\author{
Solvent consumption optimization in a lithographic process using \\ DMAIC six sigma program
}

\author{
Néstor Caicedo Solano \\ Magíster en Ciencias de la Ingeniería Industrial, Profesor Asociado, \\ Universidad Autónoma del Caribe, Grupo Optima. \\ nestor.caicedo@uac.edu.co
}

Recibido 14/08/13, Aceptado 13/10/2013

\begin{abstract}
RESUMEN
Este artículo presenta el uso de la metodología DMAMC de programas seis sigmas de calidad para disminuir consumos de disolvente químico Lule 10 utilizado en procesos de limpieza de equipos especializados para litografía sobre hojalata. El objetivo fue utilizar la metodología DMAMC como modelo para el desarrollo sostenible minimizando el impacto en sus componentes: medio ambiente, recurso humano y costos de producción; mejorando los niveles de calidad del material impreso en la línea de producción litográfica. Como resultado se obtuvo una reducción significativa del consumo del disolvente, entre el 19\% y 21\%, mejorando los procesos de limpieza de los equipos de la línea de producción y reduciendo los costos de producción asociados a este insumo.
\end{abstract}

Palabras clave: Calidad, Medio ambiente, Mejora, Seis Sigma, Químicos

\begin{abstract}
This paper presents the use of DMAMC methodology of Six Sigma quality programs to reduce the solvent consumption Lule 10 used in chemical cleaning processes of specialized equipment for lithography on tin. The aim was to use the DMAMC methodology as a model for sustainable development while minimizing the impact on its components: environment, human resources and production costs, improving quality levels of printed material in the production line lithography. The result was a significant reduction in the consumption of solvent, between $19 \%$ and $21 \%$, improving the cleaning process equipment of the production line, reducing the production costs associated with this input.
\end{abstract}

Keywords: Quality, Environment, Improvement, Six Sigma, Chemicals

\section{INTRODUCCIÓN}

En las empresas de litografía aún pequeñas [1], se utilizan equipos especializados, que con los cambios de corridas de producción deben ser limpiados con el fin de eliminar excesos de tinta que pueden llegar a generar productos no conformes. El uso de disolventes químicos, nace de la necesidad de que estos equipos puedan ser limpiados a un nivel que permita producir sin no conformidades luego de hacer cambios en colores de tintas de lotes de producción siguientes. El disolvente utilizado en este caso es el Lule 10. Los solventes orgánicos y sus residuos son considerados peligrosos por sus características de inflamabilidad, liposo- lubilidad y volatilidad, con liberación de vapores inflamables, tóxicos y explosivos, propiedades que posee el Lule 10.

La emisión al ambiente de vapores de algunos solventes orgánicos volátiles contribuye a la degradación de la capa de ozono como es el caso del tetracloruro de carbono y el tricloretano. Por otro lado en presencia de NOx y luz solar actúan como precursores de la formación de ozono ambiental, el cual produce efectos nocivos sobre la salud de la población y sobre el crecimiento de los vegetales, interfiriendo en la actividad fotosintética y en el metabolismo general de la planta. Al evaporarse rápidamente se concentran en espacios confinados y son absorbidos por el ser 
humano a través de la piel y por inhalación. Debido a sus propiedades liposolubles, luego de ingresar al organismo se concentran en tejidos grasos, acumulándose hasta alcanzar concentraciones que producen diversos efectos negativos para la salud, inmediatos o de largo plazo [2]. Por estas razones, se propone la utilización de la metodología DMAMC que en su definición sobre los programas seis sigma de calidad busca Definir, Medir, Analizar, Mejorar y Controlar procesos, productos o servicios [3], que minimicen impactos negativos sobre los clientes.

A diferencia de otras aplicaciones, en este caso el modelo DMAMC se muestra como una opción para la gestión ambiental y el desarrollo sostenible [3], tratando de minimizar el impacto en tres factores importantes:

- La salud del recurso humano que está en contacto directa o indirectamente con el proceso litográfico y de limpieza de los equipos y por ende con el disolvente.

- $\quad$ El medio ambiente que se afecta por las características químicas del producto.

- Por los costos asociados a la reducción de los consumos del disolvente.

Por estas razones, el modelo propuesto organiza metodológicamente un proyecto que impacta positivamente en los componentes del desarrollo sostenible, obteniendo resultados que evidencian una mejora significativa de los procesos y su gestión de calidad.

\section{METODOLOGÍA}

Tal como se han desarrollado procesos de optimización de consumo de productos altamente nocivos para el medio ambiente [1], el desarrollo de este trabajo estuvo enmarcado en la metodología propia DMAMC de los programas seis sigma, alineando las actividades de definir, medir, analizar, mejorar y controlar el problema, buscando alternativas estadísticas y administrativas especificas que permitieran obtener resultados satisfactorios acordes con los objetivos propuestos. La metodología que se propuso en este sentido sigue descrita a continuación [4]:

Iniciando con las primeras etapas del modelo DMAMC, definir y medir, se identificaron las variables que afectaban significativamente el alto consumo de este disolvente, reconociendo la caracterización de los procedimientos que la compañía tenía enmarcados en su sistema de gestión de calidad certificado para el uso del disolvente en las actividades de limpieza.

Estas actividades propias del proceso litográfico para la fabricación de envases de hojalata y sus indicadores, se evidenciaron a través de la observación directa sobre los registros de los departamentos de producción, gestión ambiental, despacho, control de calidad y salud ocupacional.
La obtención de información del disolvente fue importante, y para ello se revisaron las fichas técnicas y hojas de seguridad del producto, con el fin de caracterizar su aplicación y manipulación dentro del proceso litográfico de la empresa, para adoptar métodos de trabajo adecuados, buscando la protección de la salud y del medio ambiente, siguiendo las recomendaciones de la ingeniería de calidad en proyectos seis sigma $[5,6]$.

Luego se procedió al desarrollo de las siguientes fases: analizar y mejorar. En esta fase se evaluaron factores relevantes, utilizando la técnica diagrama de Pareto para priorizarlos y luego evaluarlos estadísticamente por medio del diseño experimental. Luego, diseñando y ejecutando estrategias experimentales, se lograron establecer niveles de optimización de los factores, construyendo un modelo matemático representativo del consumo.

Por último se llegó a la fase controlar; donde se diseñó y documentó un plan de manejo del disolvente a partir de la evaluación del modelo matemático propuesto anteriormente, utilizando el software MINITAB ${ }^{\circledR}$ como herramienta informática de análisis de datos, soportado en pruebas de hipótesis que evidenciaron los cambios en los consumos y los gráficos de control de consumos implementados en la empresa, así como también, un análisis de costo/beneficio a partir de la caracterización de los nuevos parámetros de manipulación y uso del disolvente.

\subsection{Técnica Gráfica de Pareto}

Con el diagrama de pareto de la figura 1, se consideraron los factores que incidían en los consumos de disolvente.

Figura 1. Gráfica de Pareto según consumos.

Figure 1. Chart for about consumptions.

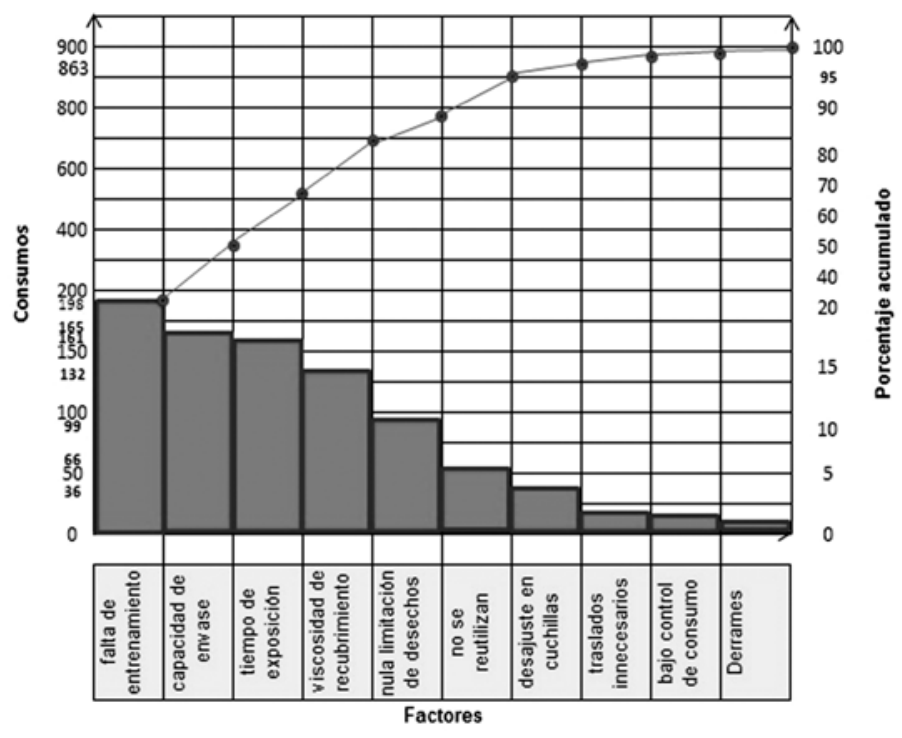


En la figura 1, se observa cómo un $20 \%$ de los factores (falta de entrenamiento, capacidad de envases, tiempo de exposición y viscosidad de los recubrimientos) representan un $80 \%$ sobre las razones de consumo de disolvente, por lo que se orientó el experimento hacia estos cuatro factores, identificando la posibilidad de reducirlo en un $80 \%$, teniendo en cuenta la importancia de cada factor de acuerdo al problema abordado [7].

\section{RESULTADOS}

\subsection{Diseño de experimento}

De una forma sistemática se diseñaron los experimentos que permitieron conocer los efectos sobre los consumos, representados como variable de respuesta. Para la ejecución del experimento, se codificaron los factores principales asociados a los consumos con sus niveles bajos y altos como - 1 y 1 respectivamente [8], dichos factores se describen a continuación:

- Viscosidades de los recubrimientos (A): bajas y altas $(-1,1)$.

- Entrenamiento del personal (B): no capacitado y capacitado. $(-1,1)$.

- $\quad$ Capacidad de envases (C): volumen de 3 y 5 galones. $(-1,1)$.

- Tiempo de exposición (D): nocturno y diurno $(-1,1)$.

El cálculo de la réplica generó 48 muestras, extraído de las curvas características y los planes de las funciones de MINITABß.

Del experimento inicial se obtuvo el siguiente modelo:

\section{$\mathrm{S}=0,381881$}

R-cuad. $=79,08 \%$ R-cuad. (Pred.) $=52,94 \%$ R-cuad. (Ajustado) $=69,28 \%$

De esta forma, podemos expresar parcialmente el modelo matemático de la siguiente forma:

$\mathrm{Y}=3.5625+0.3542 \mathrm{~A}-0.2917 \mathrm{~B}+0.2708 \mathrm{C}-0.1042 \mathrm{D}+$ $0.0000 \mathrm{AB}-0.0625 \mathrm{AC}+0.0208 \mathrm{AD}+0.1667 \mathrm{BC}-0.0417 \mathrm{BD}$ $-0.1042 \mathrm{CD}-0.0833 \mathrm{ABC}-0.0000 \mathrm{ABD}+0.0625 \mathrm{ACD}+$ $0.1250 \mathrm{BCD}-0.0417 \mathrm{ABCD}$

\subsection{Obtención del modelo reducido}

Dentro del diseño de análisis factorial, era importante identificar los términos significativos, para el modelo matemático. Para esto se hicieron pruebas de hipótesis que determinaron esta condición.
Figura 2. Hipótesis para coeficientes.

Figure 2. Test of hipothesis.

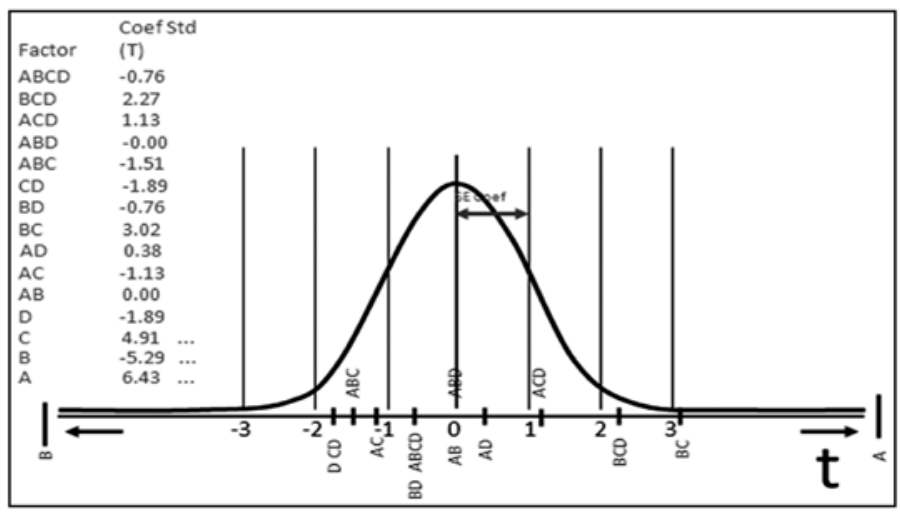

Según la figura 2, se identificaron los factores significativos A, B, C y dos interacciones. De esta forma se obtuvo una reducción del modelo matemático, a partir de los factores significativos. Desde el MINITAB ${ }^{\circledR}$ obtuvimos:

Ajuste factorial: Consumo vs. A; B; C; D

$\mathrm{S}=0,385193$ PRESS $=8,33789$

R-cuad. $=72,74 \%$ R-cuad. (Pred.) $=62,63 \%$ R-cuad. (Ajustado) $=68,75 \%$

El modelo matemático reducido quedaría de la siguiente forma:

$\mathrm{Y}=3.5625+0.3542 \mathrm{~A}-0.2917 \mathrm{~B}+0.2708 \mathrm{C}+0.1667 \mathrm{BC}+$ $0.1250 \mathrm{BCD}$

De la gráfica de Pareto en la figura 1 y los efectos estandarizados ilustrados en la figura 3 , se concluyó que el factor D no es significativo con $\alpha=0.05$, por lo tanto se omite del modelo matemático.

Figura 3. Efectos estandarizados.

Figure 3. Standarized effects.

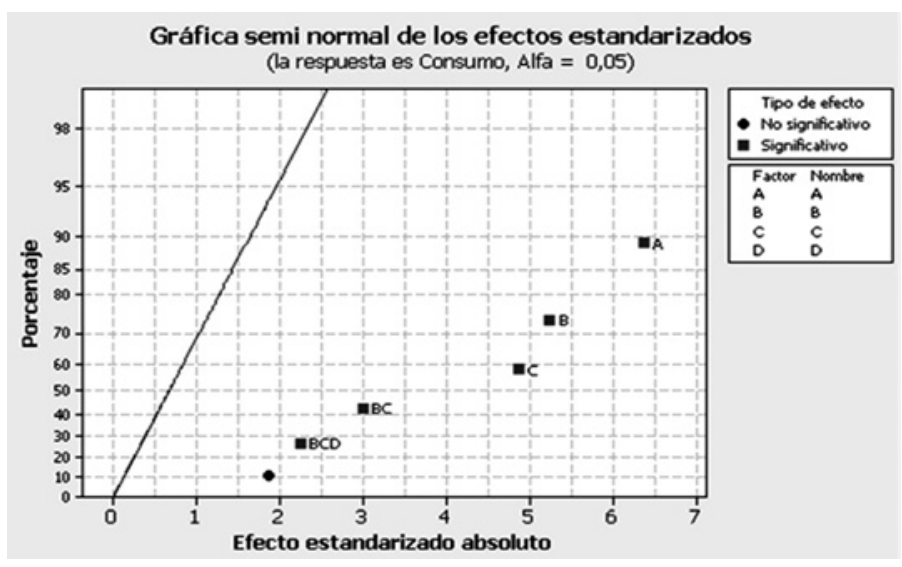


Ya que la intención era optimizar el uso de este disolvente a través de una disminución de su consumo del modelo matemático reducido, se tomaron muestras que evidencian la estimación realizada por el modelo matemático. Para esto se diseño un plan de muestreo conservando distribución normal teniendo en cuenta los niveles bajos (-1) para cada factor e iteración, obteniendo los siguientes consumos: de nuestro valor esperado 3.5 y minimizado en 3.27 galones para el consumo de disolvente en el lavado de equipos y piezas mecánicas según el modelo matemático, de lo cual se pudo obtener un promedio de $2.9687 \approx 3$ galones de consumo, una vez tomada dos replicas de 16 datos cada una. Para la comprobación de la evidente disminución de los consumos se procedió a plantear una prueba de hipótesis en la tabla 1, para verificar si los nuevos datos se ajustaban al modelo matemático.

Tabla 1. Prueba z para medias de dos muestras [9].

Table 1. Test for means differences [9].

\begin{tabular}{|l|c|c|}
\hline \multicolumn{1}{|c|}{ Parámetros } & $\begin{array}{c}\text { Consumos } \\
\text { antes (gal) }\end{array}$ & $\begin{array}{c}\text { Consumos } \\
\text { después (gal) }\end{array}$ \\
\hline Media & 3,625 & 2,96875 \\
\hline Varianza (conocida) & 0,72 & 0,49 \\
\hline Observaciones & 32 & 32 \\
\hline Diferencia hipotética de las medias & 0 & \\
\hline $\mathrm{Z}$ & 3,374827819 & \\
\hline $\mathrm{P}(\mathrm{Z}<=\mathrm{z})$ una cola & 0,000369309 & \\
\hline Valor crítico de $\mathrm{z}$ (una cola) & 1,644853627 & \\
\hline Valor crítico de $\mathrm{z}$ (dos colas) & 0,000738619 & \\
\hline Valor crítico de $\mathrm{z}$ (dos colas) & 1,959963985 & \\
\hline
\end{tabular}

Fuente. Elaboración Propia.

A partir del análisis, con un nivel de confianza del 95\% no existía suficiencia estadística para aceptar $(\mathrm{H} 0)$, por lo tanto se podía comprobar que los consumos anteriores al proyecto eran evidentemente mayores que los nuevos.

\subsection{Plan de manejo para el consumo de disolvente}

Representando de una forma gráfica la secuencia en que quedarían las actividades según el modelo matemático, se propuso un plan de manejo ilustrado en el siguiente flujograma, figura 4, que ayuda a identificar claramente las actividades, a partir de la caracterización de los nuevos parámetros de manipulación y uso, teniendo en cuenta que el problema de consumo se debía también al proceso de limpieza que requería el uso de este insumo. Este nuevo proceso se implementó y de allí se hicieron los cálculos de costos y consumos del disolvente que se muestran posteriormente.
Figura 4. Flujograma para el consumo de disolvente.

Figure 4. Flowchart for solvent consumption.

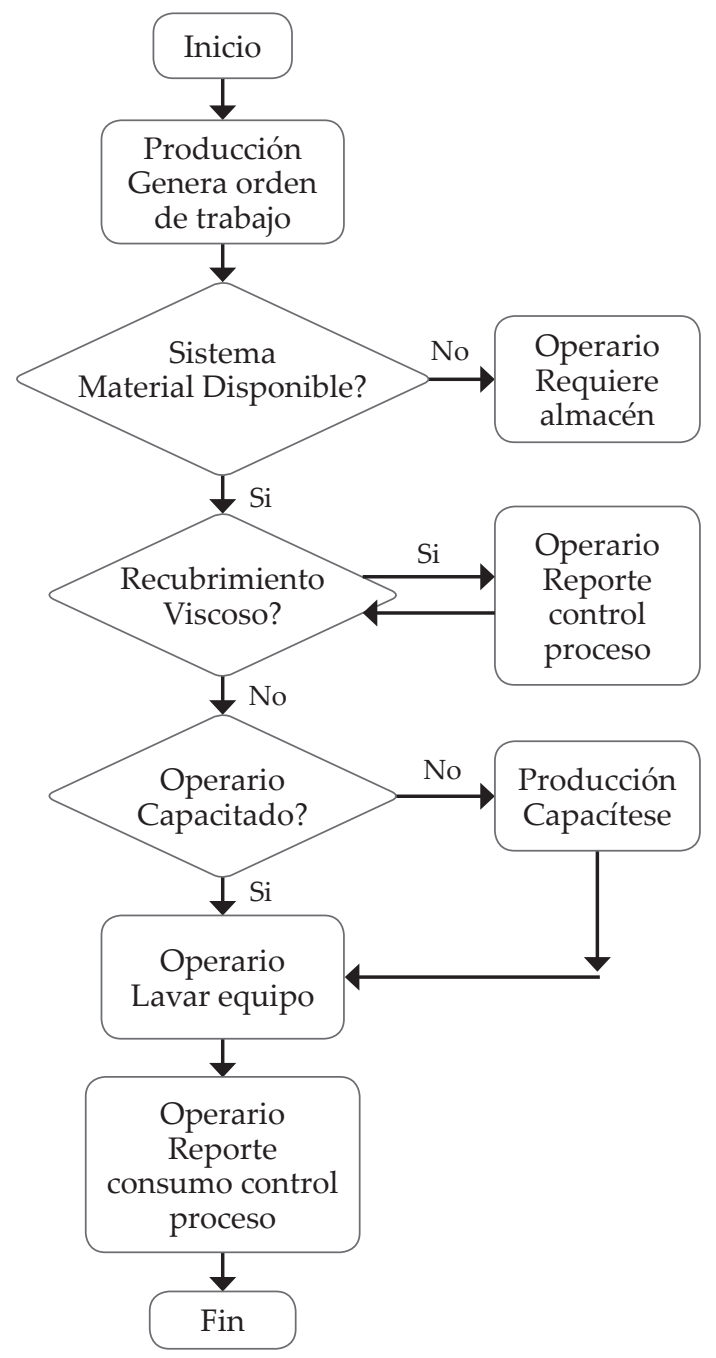

\subsection{Control estadístico de procesos}

Esta herramienta estadística fue propuesta para controlar el consumo de este disolvente pretendiendo cubrir los objetivos orientados al seguimiento y vigilancia del proceso, reducción de la variación y menores costos por consumo.

De esta forma se procedió a la construcción de los gráficos de control [10], desde Microsoft Excel ${ }^{\circledR}$, buscando ilustrar las diferencias de los consumos del disolvente antes, según la figura 5 y después de las mejoras, según la figura 6, resaltando el hecho de que el Límite Superior de Control (LSC) y el Límite de Inferior de Control (LIC), terminan indicando que luego de la mejora, el consumo está bajo control y estandarizado desde la naturaleza misma del proceso. 
Figura 5. Gráfico de control para los datos antes de la mejora. Figure 5. Control chart before improvement.

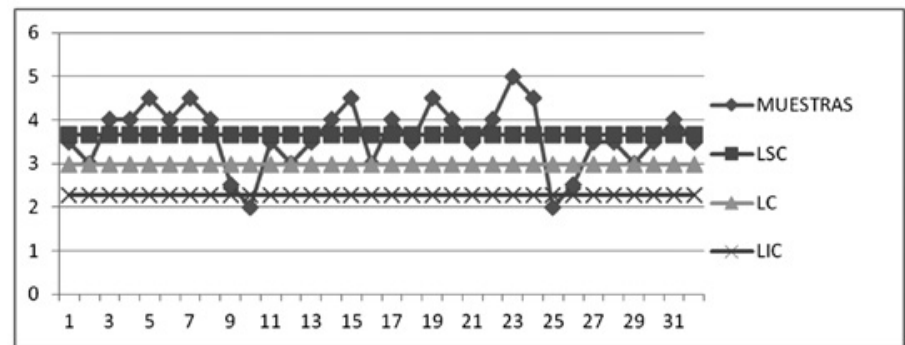

Figura 6. Gráfico de control para los datos después de la mejora.

Figure 6. Control chart after improvement.

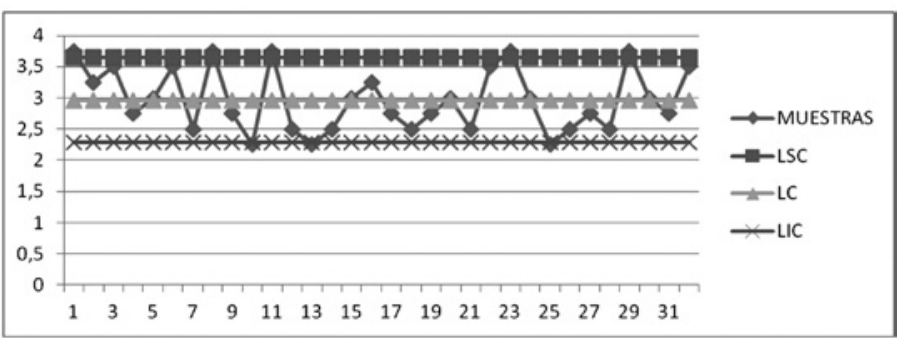

La capacidad de procesos, se utilizó como indicador de calidad [11], teniendo en cuenta que se definieron los nuevos valores de consumo según la mejora, por tanto, el valor de la capacidad de proceso indicará la capacidad del proceso de cumplir con estos nuevos valores definidos como especificación de consumo del disolvente. De esta forma se ilustra el cambio en el proceso de limpieza y consumo del disolvente, pasando de una capacidad de procesos de $\mathrm{Cp}=0.38$ a un $\mathrm{Cp}$ $=1.02$, teniendo en cuenta que los consumos máximos de la tolerancia fueron los cálculados por el proceso de optimización a través del modelo matemático. Este indicador de capacidad de proceso evidencia la mejora de un proceso antes y después de la implementación de un proyecto de mejora [12].

\subsection{Análisis costo - beneficio}

Dado que es una técnica importante dentro del ámbito de la teoría de la decisión y la administración [13], se determinó valorar la conveniencia de los cambios en términos económicos derivados de los costos y beneficios deseables e indispensables, para lo cual se resume en la tabla 2, un modelo sencillo del costo de producción del consumo de disolvente, en la tabla 3 teniendo en cuenta los factores considerados importantes según el análisis de Pareto y la comparación de los costos antes y después de la mejora, que ilustra la tabla 4.

Tabla 2. Costos de producción consumo de disolvente.

Table 2. Production costs consumption.

\begin{tabular}{|l|c|c|c|}
\hline \multicolumn{4}{|c|}{ Costos de producción } \\
\hline Lista de costos & $\begin{array}{c}\text { Consumo } \\
\text { pro/mes (2011) }\end{array}$ & $\begin{array}{c}\text { Costo } \\
\text { unitario(\$) }\end{array}$ & Costo total(\$) \\
\hline Paros de producción & 0,35 tambores & 1100000 & 385000 \\
\hline Cambios de referencias & 23,56 tambores & 1100000 & 25920000 \\
\hline Mantenimiento inventario & 24 tambores & 165000 & 165000 \\
\hline TOTAL & & & $\mathbf{2 6 4 7 0 0 0 0}$ \\
\hline
\end{tabular}

Tabla 3. Costos consumo de factores relevantes según Pareto.

Table 3. Costs consumption from Pareto diagram.

\begin{tabular}{|c|c|c|c|c|c|c|}
\hline \multicolumn{6}{|c|}{ CONSUMO DE FACTORES RELEVANTES SEGÚN PARETO ANTES DE LA MEJORA } \\
\hline Factores & $\begin{array}{c}\text { Consumos } \\
\text { (GAL) }\end{array}$ & $\begin{array}{c}\text { Costo } \\
\text { Unitario(\$) }\end{array}$ & Costo Total & $\begin{array}{c}\text { Costo } \\
\text { Acumulado }\end{array}$ & $\mathbf{\%}$ & \%Acudo. \\
\hline Falta de entrenamiento & 198 & 20000 & 3960000 & 3960000 & 22,93 & 22,93 \\
\hline Capacidad de envase & 165 & 20000 & 3300000 & 7260000 & 19,1 & 42,03 \\
\hline Tiempo de exposición & 161,44 & 20000 & 3228800 & 10488800 & 18,69 & 60,72 \\
\hline Viscosidad & 132 & 20000 & 2640000 & 13128800 & 15,28 & 76,01 \\
\hline TOTAL & $\mathbf{6 5 6 , 4 4}$ & $\mathbf{2 0 0 0 0}$ & $\mathbf{1 3 1 2 8 8 0 0}$ & & $\mathbf{7 6}$ & \\
\hline
\end{tabular}


Tabla 4. Costo diferencia de consumo antes y después.

Table 4. Cost consumptions after and before.

\begin{tabular}{|c|c|c|c|c|c|c|}
\hline \multicolumn{7}{|c|}{ DIFERENCIA ANTES Y DESPUÉS ENTRE CONSUMOS Y COSTOS } \\
\hline FACTORES & $\begin{array}{c}\text { CONSUMOS } \\
\text { (GAL) }\end{array}$ & $\begin{array}{c}\text { COSTO } \\
\text { UNITARIO(\$) }\end{array}$ & $\begin{array}{c}\text { COSTO } \\
\text { TOTAL }\end{array}$ & $\begin{array}{c}\text { COSTO } \\
\text { ACUMULADO }\end{array}$ & \% & \%ACUDO. \\
\hline Falta de entrenamiento & 33 & 20000 & 660000 & 660000 & 22,93 & 22,93 \\
\hline Capacidad de envase & 10,56 & 20000 & 211200 & 871200 & 19,1 & 42,03 \\
\hline Tiempo de exposición & 40,36 & 20000 & 807200 & 1678400 & 18,69 & 60,72 \\
\hline Viscosidad & 22,44 & 20000 & 448800 & 2127200 & 15,28 & 76,01 \\
\hline TOTAL & $\mathbf{1 0 6 , 3 6}$ & $\mathbf{2 0 0 0 0}$ & $\mathbf{2 1 2 7 2 0 0}$ & & 76 & \\
\hline
\end{tabular}

Según los resultados obtenidos de la tabla 4 , se evidencia un ahorro en los costos de consumo del disolvente después de la mejora, que fueron de \$2,127, 200/mes ó \$25, 526,400/año.

En la tabla 5 se muestra la importancia de la inversión teniendo en cuenta la relación costo-beneficio de la implementación de las mejoras realizadas al proceso.

Tabla 5. Relación costo-beneficio.

Table 5. Benefits - costs.

\begin{tabular}{|c|c|c|c|}
\hline \multicolumn{2}{|c|}{ COSTOS DE PRODUCTO DE CALIDAD } & \multicolumn{2}{c|}{ BENEFICIOS DE PRODUCTO DE CALIDAD } \\
\hline Costos & Costo unitario/mes(\$) & Beneficios & Costo unitario/mes(\$) \\
\hline Capacitación & 375000 & Capacitación & 660000 \\
\hline Tiempo Exposición & 110000 & Tiempo exposición & 807200 \\
\hline Viscosidad recubrimiento & 108800 & Viscosidad recubrimiento & 448800 \\
\hline Desechos (estopas) & 87000 & Desechos (estopas) & 2500 \\
\hline No se reutilizan & 21600 & No se reutilizan & 21600 \\
\hline Cuchillas & 56000 & Cuchillas & 56000 \\
\hline Control consumo & 108800 & Derrames & 11250 \\
\hline & & Control de consumo & 40000 \\
\hline COSTO TOTAL & $\mathbf{8 6 7 2 0 0}$ & Control consumo & 108800 \\
\hline
\end{tabular}

Se analizaron los cálculos de costo-beneficio, y se concluyó que, por cada peso gastado se obtenía un \$2.48 de retorno positivo.

\section{CONCLUSIONES}

- La importancia de abordar los programas seis sigma, facilita el proceso metodológico de implementar un proyecto de mejora de calidad en una compañía. En este caso las funciones de definir y medir el problema fueron fundamentales para la mejora significativa del consumo del disolvente y de los ahorros logrados.

- Una vez obtenido los factores causantes del alto consumo de este disolvente, se procedió al desarrollo de las siguientes fases, analizar y mejorar, utilizando herramientas como la gráfica de Pareto, que representó el grado de importancia o peso que tienen estos factores. Observándose como el $20 \%$ de los factores (falta de entrenamiento, capacidad de envases, tiempo de exposición y viscosidad de los recubrimientos) representan un $80 \%$ de los defectos por consumo de disolvente, por lo que si centrábamos el diseño de experimento en estos cuatro factores, podríamos reducirlo en un $80 \%$.

- Para el diseño y ejecución de estrategias, como todas las posibles combinaciones entre estos factores cada uno con dos niveles de significancia, se obtuvo la construcción de un modelo matemático que permitió mejorar, minimizando el consumo de este disolvente, logrando pasar de 3.62 a 2.96 galones aproximadamente.

- Se planteó una prueba de hipótesis donde se comprobó estadísticamente que los consumos después de la 
mejora realmente fueron menores con la implementación de la metodología.

- Se propuso un flujograma de procesos para la manipulación y uso del disolvente, el cual se implementó y validó la mejora y la reducción de los costos.

- Se logró demostrar a través de los gráficos de control, que el proceso anterior generaba alta variabilidad de los consumos, mientras que después de la mejora se demostró que los datos de consumo estaban dentro de los límites y el proceso se encontraba controlado.

- Con el análisis costo-beneficio, se determinó la valoración y conveniencia en términos económicos, con una relación de $\$ 2.48$ de beneficio por cada $\$ 1$ invertido con un beneficio hasta de $\$ 25,526,400$ en el primer año.

- En general el artículo muestra el uso de la metodología DMAMC asociada a los programas seis sigma, que buscan desarrollar un proyecto de mejora a través de una secuencia lógica apoyada en el análisis estadístico de datos. Desarrollar esta metodología como modelo de gestión ambiental y desarrollo sostenible quedará entonces propuesto como alternativa de mejora de calidad integral, teniendo en cuenta la relación de los sistemas integrados de gestión.

\section{REFERENCIAS}

[1] Antony, J., Kumar, M., Tiwari, M., An application of Six Sigma methodology to reduce the engine-overheating problem in an automotive company, Proceeding of the Institution of Mechanical Engineers, pp. 633-644, 2005.

[2] Frings, G., Grant, L., Who moved My Sigma...Effective Implementation of the Six Sigma Methodology to Hospitals. Quality \& Reliability Engineering International, pp. 311-328, 2005.

[3] Habidin, N. F., Yusof, S. M., Relationship between lean six sigma, environmental management systems, and organizational performance in the Malaysian automotive industry, International Journal of Automotive Technology, 13.7, 1119-1125, 2012.

[4] Daphnia, Boletín informativo sobre la prevención y la producción más limpia, 2004. Disolventes orgánicos. Programa salud ambiente y trabajo. Referencia citada de www. ccoo.es/daphnia.htm. Disponible desde: http://web.idrc.ca/ uploads/user-S/11437600891gr-02_10-solventes_pag83-88. pdf. [Acceso 5 de septiembre 2011].

[5] Bañuelas, R., Anthony, J., Brace, M., An Application of Six Sigma to Reduce Waste, Quality \& Reliability Engineering International, pp. 553-570, 2005.

[6] Kumar, M., Antony, J., Madu, C. N., Six Sigma in smalland medium-sized UK manufacturing enterprises, International Journal of Quality and Reliability Management, 22(8), 860-874, 2005.

[7] Brady, J., Allen, T., Six Sigma Literature: A Review and Agenda for Future Research, Quality and Reliability Engineering International, 22, 335-367, 2009.

[8] Montgomery D. C., Design of experiments, Wiley, New York, 2009.

[9] Montgomery D. C., Probabilidad y estadística aplicada a la ingeniería, Limusa, México, 2007.

[10] Besterfield, D., Control de Calidad, Pearson, México, 2009.

[11] Montgomery D. C., Control Estadístico de la Calidad, Wiley, New York, 2006.

[12] Pyzdek, T., The Six Sigma Project Planner: A Step-byStep Guide to Leading a Six Sigma Project through DMAMC, McGraw-Hill, 2003.

[13] Hayler, R., Nichols, M., What is Six Sigma Process Management?, McGraw-Hill, 2009. 